\title{
Development of a digital model of the working process of a hydraulic excavator
}

\author{
Rustem Nabiullin, Anatoliy Komissarov, and Viktor Shestakov \\ Ural State Mining University, 620144 Ekaterinburg, Russia
}

\begin{abstract}
In this work, we consider a method for determining the rational values of the structural and operational parameters of the lever - hydraulic mechanisms of hydraulic excavators based on an analysis of the results of a computational experiment obtained at the output of a mathematical model of a workflow. It is shown that the presence of kinematic connections between the engine (hydraulic cylinder) and the links of the lever-hydraulic mechanism causes a change in the relationship between the operating parameters of the engine and the power parameters implemented on the driven members (bucket, handle and arrow), depending on the geometric parameters (lengths) of links and coordinates of points (axes) connections links. A simulation model of the working process is developed, which allows to determine the operational parameters of the lever - hydraulic mechanisms.
\end{abstract}

\section{Introduction}

Lever mechanisms differ from other mechanisms in that they have "individual" kinematic properties, which are determined by the structural diagram of the mechanism, the type of kinematic connections between the links and the geometric parameters (lengths) of the links.

The operating parameters of hydraulic cylinders that are part of the executive lever hydraulic mechanisms of a hydraulic excavator (boom rotation mechanism, handle rotation mechanism and bucket rotation mechanism) are converted into slave link parameters during operation in accordance with the properties of the lever mechanisms. The main characteristic of the lever mechanism is the kinematic and dynamic transfer functions (gear ratios), which determine the relationships between the kinematic and dynamic parameters of the driven and leading links.

The difficulty in ensuring the required digging efforts and maintaining the stability of the operating parameters of the working equipment of hydraulic excavators during the digging cycle is due to the variation in the gear ratios. In addition, during the excavation of rock mass on the hydraulic cylinders for excavator boom rotation and handle rotation, which are in the "closed" (passive) state, the force of resistance to digging acts, which leads to the appearance of significant reactive loads (exceeding the magnitude of the forces developed by the hydraulic cylinders in the active mode) .

The purpose of the study - is to identify reserves for increasing the reliability and efficiency of the working equipment of hydraulic excavators. 


\section{Research Objectives:}

- Definition of kinematic and dynamic transfer functions that determine the operating parameters of the working equipment of hydraulic excavators;

- Definition of active and reactive loads acting on the engines (hydraulic cylinders) of lever-hydraulic mechanisms;

- Definition of the operating characteristics of the excavator from the conditions for ensuring the required digging efforts and excluding the operation of safety valves.

\section{The solution of research problems}

The noted features show that the methodology for determining the parameters of working equipment must take into account a sufficiently large number of influencing factors, which can be implemented using mathematical models for calculating kinematic and power parameters with their implementation in an algorithmic language for computers [1]. This allows at the design stage to conduct studies of the influence of the operational parameters of the mechanisms of the working equipment on the parameters of the driven links forming the output functions of the system.

The technique should perform several functions:

- determine the geometric parameters of the working equipment and the dimensions of the hydraulic cylinders according to the condition of the formation of the working area of the excavator for the given digging efforts;

- determine the possible digging efforts within the working area;

- determine the efforts on the rods of hydraulic cylinders and other elements of the working equipment according to the geometric parameters of the working equipment, the position of the bucket and the specified digging force;

- to determine the stresses in the elements of the working equipment with given geometric dimensions and values of forces in the elements.

The dependencies included in the methodology for calculating the parameters will be determined by the constructive scheme of the excavator. Fig. 1 shows a diagram of an excavator with working equipment "backhoe".

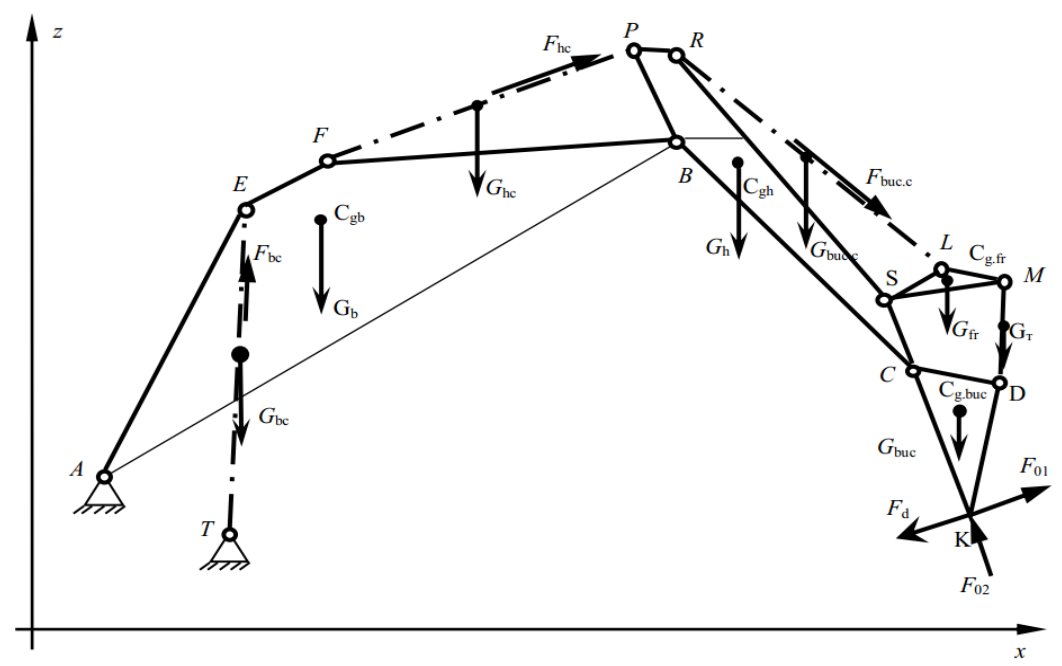

Fig. 1. Diagram of working equipment. 
$\mathrm{G}_{\mathrm{b}}, \mathrm{G}_{\mathrm{h}}, \mathrm{G}_{\mathrm{buc}}, \mathrm{G}_{\mathrm{bc}}, \mathrm{G}_{\mathrm{hc}}, \mathrm{G}_{\mathrm{buc} . \mathrm{c}}, \mathrm{G}_{\mathrm{fr}}, \mathrm{G}_{\mathrm{T}},-$ gravity of the boom, handle, bucket, boom cylinders, handle, bucket, frame and traction; $\mathrm{C}_{\mathrm{gb}}, \mathrm{C}_{\mathrm{gh}}, \mathrm{C}_{\mathrm{g} . \mathrm{buc}}, \mathrm{C}_{\mathrm{g} . \mathrm{fr}}$ - centers of gravity of an boom, handle, bucket, frame; $F_{b c}, F_{h c}, F_{b u c . c}$ - design forces on the cylinder rods of the boom, handle, bucket; $F_{01}, F_{02}$ tangent and normal components of digging resistance

The initial data for the calculations are the linear dimensions of the elements of the working equipment between the characteristic points (hinges, centers of mass), the masses of the elements, the forces on the rods of the hydraulic cylinders, corresponding to the setting of the safety valves. These values are entered as initial data for the stress calculation program at all possible points of the working area. According to the values of the efforts on the elements of the working equipment, the design of the boom, stick, bucket and hydraulic cylinders is performed. Based on the masses of elements specified during the design, the recalculation of digging forces from the condition for excluding the operation of the safety valves is carried out, as well as the calculation of the required pressures for the hydraulic cylinders.

At the initial stage, a kinematic and power analysis of the lever - hydraulic mechanisms was performed.

In the general case, the expression for the kinematic transfer function of the lever hydraulic mechanism has the form [2]:

$$
\Phi_{v}=\frac{V_{\text {Out.link. }}}{V_{\text {rod }}}=f\left(l_{i}, \alpha_{i}, X_{\text {out.link. }}, \mathrm{y}_{\text {out.link. }}\right)
$$

Where $V_{\text {Out-link }}, V_{\text {rod }}$ - the speed of the characteristic point of the output link and the speed of the rod, respectively; $l_{i}$ - link length; $\alpha_{i}$ - angular distances between links; $X_{\text {outlink }}$ и $Y_{\text {outllink }}$ - coordinates of the characteristic point of the output link.

In hydraulic excavators, the main (digging) mechanism is the bucket rotation mechanism, and the forces on the cutting edge of the bucket $F_{\mathrm{k}}$ are determined by the power transfer function of the bucket rotation mechanism, which has the following form and represents the relationship between the power parameters implemented at the output and driving links of the mechanisms:

$$
\Phi_{F_{b u c, c}}=\frac{F_{d}}{F_{\text {buc } . c}} ;
$$

Where $F_{\text {buc.c }}$ - force acting on the rod of the hydraulic cylinder of the bucket rotation mechanism; $F_{\mathrm{d}}$ - force acting on the cutting edge (teeth) of the bucket. form:

For the boom and handle rotation mechanisms, the power transfer functions have the

$$
\begin{aligned}
& \Phi_{T_{\text {hand.cyl. }}}=\frac{T_{\text {hand. }}}{F_{\text {he }}} ; \\
& \Phi_{T_{\text {boom.cyl. }}}=\frac{T_{\text {boom. }}}{F_{b c}} ;
\end{aligned}
$$

Where $\mathrm{F}_{\mathrm{hc}}, \mathrm{F}_{\mathrm{bc}}$ - forces acting on the rods of hydraulic cylinders of the handle and boom rotation mechanisms; $T_{\text {hand., }} T_{\text {boom. }}-$ forces torque $\mathrm{F}_{\mathrm{hc}} \mathrm{uF}_{\mathrm{bc}}$ relative to axes $\mathrm{B}$ and $\mathrm{A}$.

Instability of active loads during the excavation cycle leads to a change in reactive loads.

Maximum reactive loads occur with an unfavorable combination of the following factors:

- at the maximum (or close to it) value of the shoulder, the resistance forces of the rock to digging from the axes $\mathrm{A}$ or $\mathrm{B}$;

- the maximum (or close to it) value of the power transfer function of the bucket rotation mechanism; 
- at the minimum (or close to it) value of the power transfer function of the boom rotation mechanism or handle rotation mechanism;

- if the directions (or small deviation) of the action of the gravity of the elements of the working equipment and the resistance of the rock to digging (tangent component) coincide.

To assess the magnitude of the reactive load, a reactive load coefficient is proposed:

$$
\begin{aligned}
& C_{\text {reac.load.boom. }}=\frac{T_{\text {ex.load.boom. }}}{F_{\mathrm{Lc}} \Phi_{T_{\text {boom.cyl. } i}}} ; \\
& C_{\text {reac.load.hand. }}=\frac{T_{\text {ex.load.hand. }}}{F_{\mathrm{Lp}} \Phi_{T_{\text {hand.cyl. } i}}} ;
\end{aligned}
$$

where $T_{\text {ex.forc.boom. }}, T_{\text {ex.forc.hand.- }}$ torque of external loads relative to the axis A and B, respectively; $\Phi_{T_{\text {boom.cyl. } i}}, \Phi_{T_{\text {hand.cyl. }}}$ - current values of the power transfer functions of the boom and stick rotation mechanism, respectively.

The operating characteristic of a hydraulic excavator determines the actual areas of the working area in which the forces on the cutting edge of the bucket are realized from the condition that the safety valve does not work under the action of reactive forces, taking into account the transfer functions of the working mechanisms.

The algorithm for calculating the boundaries of the working area and efforts at points within the zone includes three cycles and is organized as follows:

- in the external first cycle, the distance between the $T E$ points (see Fig. 1) changes by extending the boom of the hydraulic cylinders for boom rotation from the minimum (the rods of the hydraulic cylinders of the boom are fully retracted) to the maximum values (the rods of the hydraulic cylinders of the boom are fully extended);

- in the second cycle, for each distance value TE, the value of the FP segment changes from the minimum possible value (the piston rods of the handle are fully retracted) to the maximum possible value (the piston rods of the handle are fully extended);

- in the third cycle, for each value of the TE and FP distances, the value of the RL segment changes from the minimum possible value (the rods of the bucket cylinders are fully retracted) to the maximum possible value (the rods of the bucket cylinders are fully extended).

The values of the TE, FP, RL segments in the model are designated $\mathrm{L}_{b c}, \mathrm{~L}_{\mathrm{hc}}$, $\mathrm{L}_{\text {buc.c }}$ (the lengths of the boom, handl and bucket hydraulic cylinders) respectively and are used to calculate the coordinates of the working area, the characteristic points of the design scheme, the forces on the teeth of the kosh and in the elements of the working equipment.

To calculate the possible digging efforts inside the working area, the fourth cycle of searching for an acceptable solution is included in the algorithm. Before the cycle, the tangent component of the digging resistance force $F_{01}$ is specified, which is obviously greater than the possible digging force of the excavator in question. According to $F_{01}$, the forces on the rods of the hydraulic cylinders of the boom $F_{b c}$, the handle $F_{h c}$ and the bucket $\mathrm{F}_{\text {buc.c }}$ are determined.The forces on the rods are compared with the set values of the efforts of the hydraulic cylinders of the boom, handle and bucket when the safety valves are triggered. If the specified values of any of the calculated values of $F_{b c}, F_{h c}$ or $F_{b u c . c}$ are exceeded, the force $F_{01}$ decreases, and the calculations are repeated until the specified forces on the rods of all hydraulic cylinders are greater than or equal to the calculated forces.

The mathematical model and algorithm are implemented by a computer program in the algorithmic language Visual Basic for Aplication. The program implements the output of the results in tabular form and in the form of a figure, which shows on a scale the diagram of the excavator and the working area with possible digging efforts (Fig. 2). 


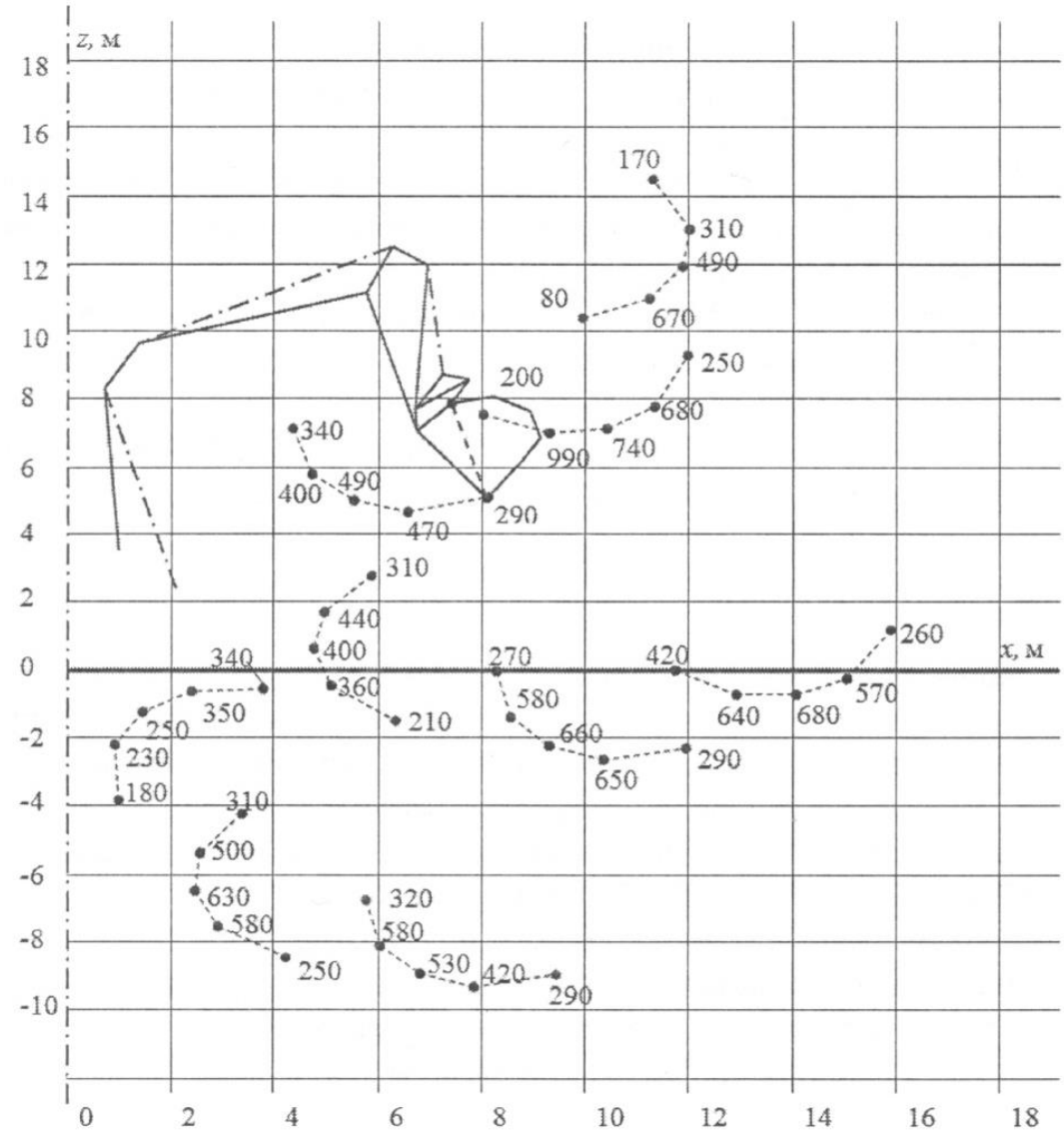

Fig. 2 Possible values of digging forces kN, within the working area.

\section{Conclusions}

The developed model of the functioning of the working equipment allows to determine the loads taking into account the parameters of the design schemes and the type of dynamic transfer functions of the lever - hydraulic mechanisms, which makes it possible to identify reserves for increasing the reliability and efficiency of the working equipment of hydraulic excavators.

\section{References}

1. P. Babenkov, V. Shestakov, Min. equip. and. electr., 10 (2018)

2. A. Komissarov, V. Shestakov, Min. equip. and. electr., 20 (2013)

3. A. Komissarov, Yu. Lagunova, V. Shestakov,Innovative engineering, 30-32(2017)

4. O.A. Lukashuk, A.P. Komissarov, Y.A. Lagunova, IOP Conference Series: Materials Science and Engineering, 709 (2), 022117 (2020)

5. P.A. Pobegailo, Mountain Information and Analytical Bulletin, 5, 107 (2016)

6. Le QuangHoan, Yang Soon-Yong, Proc. on 11th International Conference on Control, Automation and Systems, 941 (2011)

7. A.J. Koivo, M. Thoma, E. Kocaoglan, J. Andrade-Cetto, Journal of Aerospace 
Engineering, 9, 10 (1996)

8. D.A. Bradley, D.W. Seward, J.E. Mann, M.R. Goodwin, Automation in Construction, 2(3), 217 (1993)

9. P.A. Pobegailo, Mountain Information and Analytical Bulletin, 5, 212 (2015) 